\title{
Super-resolution study of PIAS SUMO E3-ligases in hippocampal and cortical neurons
}

\author{
Andrea Conz, ${ }^{1}$ Clara Alice Musi, ${ }^{2,3}$ Luca Russo, ${ }^{1}$ Tiziana Borsello, ${ }^{2,3}$ Luca Colnaghi ${ }^{1}$ \\ ${ }^{1}$ Department of Molecular Biochemistry and Pharmacology, Istituto di Ricerche Farmacologiche Mario Negri IRCCS, \\ Milan; \\ ${ }^{2}$ Department of Neuroscience, Istituto di Ricerche Farmacologiche Mario Negri IRCCS, Milan; \\ ${ }^{3}$ Department of Pharmacological and Biomolecular Sciences, University of Milan, Italy
}

\begin{abstract}
The SUMOylation machinery is a regulator of neuronal activity and synaptic plasticity. It is composed of SUMO isoforms and specialized enzymes named E1, E2 and E3 SUMO ligases. Recent studies have highlighted how SUMO isoforms and E2 enzymes localize with synaptic markers to support previous functional studies but less information is available on E3 ligases. PIAS proteins - belonging to the protein inhibitor of activated STAT (PIAS) SUMO E3-ligase family - are the best-characterized SUMO E3-ligases and have been linked to the formation of spatial memory in rodents. Whether however they exert their function co-localizing with synaptic markers is still unclear. In this study, we applied for the first time structured illumination microscopy (SIM) to PIAS ligases to investigate the co-localization of PIAS1 and PIAS3 with synaptic markers in hippocampal and cortical murine neurons. The results indicate partial co-localization of PIAS1 and PIAS3 with synaptic markers in hippocampal neurons and much rarer occurrence in cortical neurons. This is in line with previous super-resolution reports describing the co-localization with synaptic markers of other components of the SUMOylation machinery.
\end{abstract}

Key words: Neurons; SUMO; synapse; hippocampus; cortex.

Correspondence: Dr. Luca Colnaghi, Department of Molecular Biochemistry and Pharmacology, Istituto di Ricerche Farmacologiche Mario Negri IRCCS, 20156 Milan, Italy. E-mail: luca.colnaghi@marionegri.it

Contributions: AC, LC, designed the experiments; AC, CAM, conducted the experiments; AC, LR, LC, analyzed the data; AC, TB, LC, wrote/revised the paper.

Ethical approval: All procedures were approved by the Mario Negri Institute Animal Care and Use Committee, which includes ad hoc members for ethical issues, and by the Italian Ministry of Health.

Conflict of interest: The authors declare no competing interests.

Availability of data and material: The data used to support the findings of this study are available from the corresponding author on reasonable request.

Funding: This work was supported by Brightfocus Grant A2019296F and a grant from the Fondo di BeneficenzaGruppo Intesa Sanpaolo to LC. 


\section{Introduction}

In neurons post-translational modifications (PTMs) regulate the signaling cascade that guarantees an efficient, rapid and reversible response to extracellular stimuli. ${ }^{1,2}$ SUMOylation is one of the main regulators of neuronal activity ${ }^{3,4}$ and synaptic plasticity. ${ }^{5-7}$ It may also play a role in pathological processes that cause neurological disorders such as Parkinson's and Alzheimer's diseases (AD) ${ }^{4,8-10}$ SUMOylation involves the covalent conjugation of ubiquitin-like proteins, called small ubiquitin-like modifiers (or SUMO), to lysine residues of specific targets, by an enzymatic cascade. ${ }^{11}$ Five variants (SUMO1-5) have been found in mammals and brain cells very likely only express SUMO1-3. ${ }^{12,13}$ While their aminoacidic sequences differ, they share an almost identical threedimensional structure. ${ }^{14,15}$

The proteins responsible for SUMO conjugations are the SAE1/SAE2 heterodimers, known as the SUMO-E1 enzyme, the SUMO-E2 ligase Ubc9, and various SUMO-E3 enzymes. ${ }^{11}$ SUMO proteins are initially activated by SAE1/SAE2 and are subsequently transferred to Ubc9, which catalyzes the conjugation of SUMO proteins to lysine residues of the target substrates. Ubc9 can be assisted during this conjugation by SUMO-E3 enzymes such as, for example, members of the PIAS family. ${ }^{16}$

Unlike the ubiquitin system, which comprises a multitude of E3 ligases,${ }^{17}$ the SUMOylation pathway can only count on members of the PIAS family and a few other SUMO ligases (such as ZNF451 and RanBP2). ${ }^{16,18}$ SUMO can be removed from targets by specialized isopeptidases, such as members of the SENP family. ${ }^{19}$ All together, the SUMO isoforms, ligases and the SUMO proteases form the SUMOylation machinery.

Similarly to other cells, the SUMOylation machinery has nuclear and extranuclear functions in neurons. Among the extranuclear roles, it modulates synaptic activity - as first described in rat hippocampal neurons by Martin and colleagues. ${ }^{20}$ Since then, several reports have finely described how the SUMOylation machinery, in both hippocampal and cortical neurons, is required for proper neuronal functioning. ${ }^{21-23}$ Recently however, two reports have questioned the role of SUMOylation in synaptic plasticity. The authors failed to detect i) SUMOylation in synaptic fractions and ii) co-localization of SUMO1 with synaptic markers. ${ }^{24,25}$ Thus, they concluded that SUMOylation of synaptic proteins is, at best, very rare. Subsequent studies from our group employed structured illumination microscopy (SIM), a super-resolution technique that doubles the resolution of conventional microscopes, to systematically confirm the co-localization of several components of the SUMOylation machinery with both pre- and post-synaptic markers. ${ }^{26-28}$ Other groups have also pointed to new roles of SUMOylation in the physiology of synapses, ${ }^{6,29}$ thus validating the likely role of SUMOylation in synaptic plasticity. Currently however, localization studies of members of the PIAS E3 SUMO ligase family in neurons are still lacking, limiting our understating of how E3 SUMO enzymes may affect synaptic function in health and disease. This group of proteins comprises five members: PIAS1, PIAS3, PIASx $\alpha$, PIASx $\beta$, and PIAS $\gamma(\text { PIAS4) })^{30,31}$ and PIAS 1 and PIAS3 are reported to be expressed in hippocampal and cortical neurons. ${ }^{32}$

In neurons, the expression of PIAS1 is modulated by NMDA receptors and signaling pathways such as the Ras-MAP kinase (MAPK) pathway and the transcription factor cAMP response element-binding protein (CREB). ${ }^{33}$ PIAS1 SUMOylates proteins that have key roles in the formation of memory, for instance CREB itself, and its knockdown alters spatial learning. ${ }^{34}$ PIAS3 may be involved in the physiology of memory as well, since it has been implicated in the memory-related signaling of neuronal nitric oxide synthase (nNOS) and nitric oxide (NO). ${ }^{35}$ PIAS3 also direct- ly regulates gephyrin, a synaptic scaffold protein, and GABAergic transmission. ${ }^{36}$ Moreover, in hippocampal neurons, the AD-related peptide amyloid- $\beta$ (A $\beta)$ alters the expression of PIAS3 and its targets, to cause memory deficits. ${ }^{37}$ PIAS1 is also involved in the pathological effects of $A \beta$, further indicating a putative role of the SUMOylation machinery in AD. ${ }^{38}$. PIAS1 and PIAS3 are also linked to central nervous system pathologies such as Huntington disease $^{39,40}$ and schizophrenia ${ }^{41}$ and have other neuronal roles, too. For instance, PIAS1 can interact with necdin, a regulator of antiapoptotic and pro-survival processes in neurons and neural stem cells, ${ }^{42}$ and act as a biomarker of stress susceptibility, as it modifies glucocorticoid receptors ${ }^{43}$ and transcription factors able to produce stress-induced changes ${ }^{44}$ that can lead to chronic social defeat stress. $^{43}$

In view of the roles of PIAS1 and PIAS3 in physiological and pathological aspects of synaptic plasticity, we employed SIM to systematically examine their localization in hippocampal and cortical neurons, to complete the super-resolution characterization of the SUMOylation machinery in neurons. ${ }^{26,27}$

\section{Materials and Methods}

\section{Animals}

Procedures involving animals were conducted in conformity with the institutional guidelines at the Istituto di Ricerche Farmacologiche Mario Negri IRCCS, in compliance with national (Decree 26/2014; Authorization n. 19/2008-A issued March 6, 2008 by Ministry of Health) and international laws and policies (EEC Council Directive 2010/63/UE; the NIH Guide for the Care and Use of Laboratory Animals, 2011 edition). They were reviewed and approved by the Mario Negri Institute Animal Care and Use Committee, which includes ad hoc members for ethical issues, and by the Italian Ministry of Health (Decree n. 420/2017PR). Animal facilities meet international standards and are regularly checked by a certified veterinarian who is responsible for health monitoring, animal welfare supervision, experimental protocols, and review of procedures.

\section{Primary cultures}

For the preparation of primary hippocampal and cortical neurons we employed the protocol previously described by Colnaghi et al. ${ }^{26}$ Dissected hippocampi and cortexes from two-day-old CD1 mice were incubated at $37^{\circ} \mathrm{C}$ for $30 \mathrm{~min}$ in a solution of $5.8 \mathrm{mM}$ $\mathrm{MgCl}_{2}, 0.5 \mathrm{mM} \mathrm{CaCl}_{2}, 3.2 \mathrm{mM}$ HEPES, $0.2 \mathrm{mM} \mathrm{NaOH}$ (pH 7.4, $292 \mathrm{mOsm}$ ) and $20 \mathrm{U} / \mathrm{mL}$ papain (Sigma-Aldrich, St. Louis, MO, USA). The tissues were then dissociated and the single cells were plated in Ibidi micro-Slide 8-well plates to a concentration of 75,000 cells per well. Neurobasal Basal Medium (Gibco, Waltham, MA, USA) was used as medium, containing B27 (Gibco), penicillin/streptomycin and $2 \mathrm{mM}$ glutamine.

\section{Immunofluorescence experiments}

Immunofluorescence experiments followed the procedure previously described by Colnaghi et al. ${ }^{26}$ Primary neurons, after $12-14$ days in vitro (DIV) were fixed in $4 \%$ paraformaldehyde (PFA) for $15 \mathrm{~min}$. Neurons were then permeabilized with phosphate-buffered saline (PBS), $\mathrm{pH} 7.4,0.2 \%$ Triton $\mathrm{X}-100$ for $1 \mathrm{~min}$, and incubated for $1 \mathrm{~h}$ with the PBS blocking solution containing $100 \mu \mathrm{g} / \mathrm{mL}$ BSA.

To localize PIAS1 and PIAS3 proteins, neurons were incubated for $2 \mathrm{~h}$ at room temperature with primary antibodies against PIAS1 24.6 $\mu \mathrm{g} / \mathrm{mL}$ (D33A7 XP; Cell Signaling, Danvers, MA, USA) and PIAS3 $76 \mu \mathrm{g} / \mathrm{mL}$ (D5F9 XP; Cell Signaling). Co-local- 
ization studies used antibodies against Map2 19 mg/mL (AB5392; Abcam, Cambridge, UK), synaptophysin $1 \mathrm{mg} / \mathrm{mL}$ (SigmaAldrich, S5768) and PSD-95 $1 \mathrm{mg} / \mathrm{mL}$ (NeuroMabs, 75-028; Antibodies Incorporated, Davis, CA, USA). All primary antibodies were diluted in a PBS solution containing $100 \mu \mathrm{g} / \mathrm{mL}$ BSA and $0.2 \%$ Triton $\mathrm{X}-100$.

Neurons were then treated with secondary antibodies (DyLight Fluor Antibody, Thermo Fisher Scientific) for $1 \mathrm{~h}$ at room temperature, followed by incubation with nuclei-staining solution Hoechst 33342 (Thermo Fisher Scientific, Waltham, MA, USA) at $2 \mu \mathrm{g} / \mathrm{mL}$.

ProLong Glass Antifade Mountant (Thermo Fisher Scientific) was used to fix the neurons in the wells with the slides.

\section{Confocal and structured illumination microscopy}

Both the confocal images, acquired using a 40x objective, and the SIM images, acquired in 3D-SIM mode using a 100x objective, were obtained with a Nikon N-SIM microscope. Fiji software (ImageJ) was used to process the images.

\section{Statistical analysis}

We acquired forty five images from three different experiments for each condition, and analyzed the overlapping fluorescence signals with JACoP, ${ }^{45}$ a specialized toolbox for subcellular co-localization analysis under ImageJ. Pearson's correlation coefficients were calculated to indicate the overlap between the signals. Mander's M1 and M2 parameters were quantified to determine the co-localization between PIAS1 and PIAS3 with the synaptic markers synaptophysin ${ }^{46}$ or PSD- $95 .{ }^{47}$ To avoid background noise, the threshold was set manually. Graphs were drawn using GraphPad Prism 7.

\section{Results}

To determine the localization of PIAS proteins in neurons, we employed SIM $^{48}$ in cultured primary cells. Unlike conventional microscopy, SIM reaches a resolution of about $100 \mathrm{~nm}$ laterally and lends itself well suited to co-localization studies. ${ }^{48-51}$ We first determined the co-localization of PIAS1 and PIAS3 with synaptic markers in hippocampal primary neurons. ${ }^{52}$ We fixed 14-day cultured primary hippocampal neurons and incubated them with antibodies directed against PIAS1, MAP2, a neuron-specific cytoskeletal protein used as neuronal marker, and the pre- and post-synaptic markers synaptophysin ${ }^{46}$ and PSD-95. ${ }^{47}$

To examine the morphology and staining of hippocampal neurons, we acquired images using confocal microscopy with a 40x objective and confirmed the presence of PIAS1 in the nucleus and cytoplasm (Figure $1 \mathrm{~A}, \mathrm{G}$ ). To study the co-localization of PIAS1 with synaptic markers, we obtained SIM images of the neuronal processes of the cultured neurons stained with antibodies against PIAS1 and synaptophysin (Figure 1B). To determine the co-localization of the two proteins, we made a profile analysis of single loci. The results suggested that PIAS1 can colocalize with the presynaptic marker synaptophysin (Figure 1 C,D). We quantified the fraction of PIAS1 that overlaps synaptophysin (Mander's M1) and vice versa (Mander's M2) ${ }^{48}$ using $\mathrm{JaCop}^{45}$ and the results indicated low-to-medium co-localization between the pre-synaptic marker and PIAS1. Finally, we calculated the Pearson's correlation coefficient, ${ }^{53}$ which gave values suggesting partial co-localization of PIAS1 with the pre-synaptic marker (Figure 1 E,F). To verify the co-localization of PIAS1 with post-synaptic markers, we acquired images of hippocampal neurons stained with antibodies against PIAS1 and PSD-95 (Figure 1H) with SIM. Intensity profiles from loci corresponding to neuronal processes indicated that PIAS1 can localize with the post-synaptic marker (Figure $1 \mathrm{I}, \mathrm{J}$ ). We next quantified the Mander's and Pearson's correlation coefficients (Figure $1 \mathrm{~K}, \mathrm{~L}$ ). These indicated low-to-medium co-localization between PIAS1 and PSD-95. Overall, the data suggest that PIAS1 co-localizes with synaptic markers.

Next we ran a similar analysis for PIAS3 on cultured primary hippocampal neurons, staining them with antibodies directed against PIAS3, MAP2 and the pre- and post-synaptic markers synaptophysin and PSD-95. Confocal microscopy confirmed that PIAS3 is a nuclear protein than can also be found in the cytoplasm (Figure $2 \mathrm{~A}, \mathrm{G}){ }^{54}$ Although the protein is mainly nuclear, SIM (Figure 2B) and profile intensity showed co-localization between synaptophysin and PIAS3 (Figure 2 C,D). We quantified the colocalization using Mander's and Pearson's correlation coefficients (Figure 2 E,F) and found present but weak co-localization of PIAS3 and the pre-synaptic marker synaptophysin.

We then used SIM to confirm the co-localization between PIAS3 and the post-synaptic marker PSD-95 (Figure 2H), determining both the intensity profiles of the individual loci (Figure 2 I,J) and Mander's and Pearson's coefficients (Figure 2 E,F). The results describe partial co-localization between PIAS3 and the post-synaptic marker PSD-95, similarly to what we found for the pre-synaptic marker.

Having concluded the analysis on hippocampal neurons, we decided to verify whether PIAS1 and PIAS3 co-localize with synaptic markers in cortical neurons. To do this, we cultured primary cortical neurons and fixed them for microscopy. We stained them with antibodies raised against PIAS1, MAP2, synaptophysin and/or PSD-95. In images acquired using confocal microscopy with a 40x objective (Figure $3 \mathrm{~A}, \mathrm{G}$ ) PIAS1 was present more at the nuclear level than in the cytoplasm, just like in hippocampal neurons.

Next we investigated the co-localization of PIAS1 and the presynaptic marker synaptophysin with SIM (Figure 3B). The intensity profile suggested that co-localization can occur (Figure 3 C,D) but quantitative analysis, by calculating Mander's and Pearson's coefficients, indicated that the co-localization was rare (Figure 3 E,F), suggesting a difference between hippocampal and cortical neurons.

Similar analyses were conducted on cortical neurons stained with antibodies against PIAS1 and PSD-95 (Figure 3 H,I,J). Mander's and Pearson's coefficients again suggested low-to-nil colocalization between PIAS1 and PSD-95 (Figure 3 K,L). To conclude these analyses, we determined the localization of PIAS3 in cortical neurons, staining them with antibodies directed against PIAS3, MAP2 and the two pre- and/or post-synaptic markers. In these cells PIAS3 was enriched in the nucleus but barely detectable in the cytoplasm (Figure 4 A,G). SIM indicated that the co-localization was detectable (Figure 4 B-D,H-J) but it was rare (Figure 4 E-F,K-L).

\section{Discussion}

The SUMOylation machinery has been described in both hippocampal and cortical neurons in several reports. ${ }^{6,13}$ The data so far in primary hippocampal neurons are in line with previous SIM analysis, that showed partial co-localization between members of the SUMO machinery (such as SUMO1, SUMO2/3, Ubc9 and SENPs proteins) and synaptic markers. ${ }^{26,27}$ This all supports a role of the whole SUMOylation machinery in the formation of spatial memory and long-term potentiation (LTP).

In the last decade, several studies have investigated the implication of PIAS proteins in the formation of spatial memory. An increase in PIAS1 expression in hippocampal neurons of the CA1 
area is linked to better spatial learning and memory performance than in rats whose PIAS1 expression was silenced. ${ }^{32}$ Although the mechanisms underlying PIAS1-mediated memory facilitation are not fully understood, PIAS1 has been shown: i) to increase
SUMOylation of the spatial memory-related protein STAT1 (signal transducer and activator of transcription-1) $)^{32,55}$ and ii) to SUMOylate CREB. ${ }^{34}$ CREB is modified by several PTMs ${ }^{56,57}$ and its SUMOylation by PIAS1 is essential for spatial memory forma-
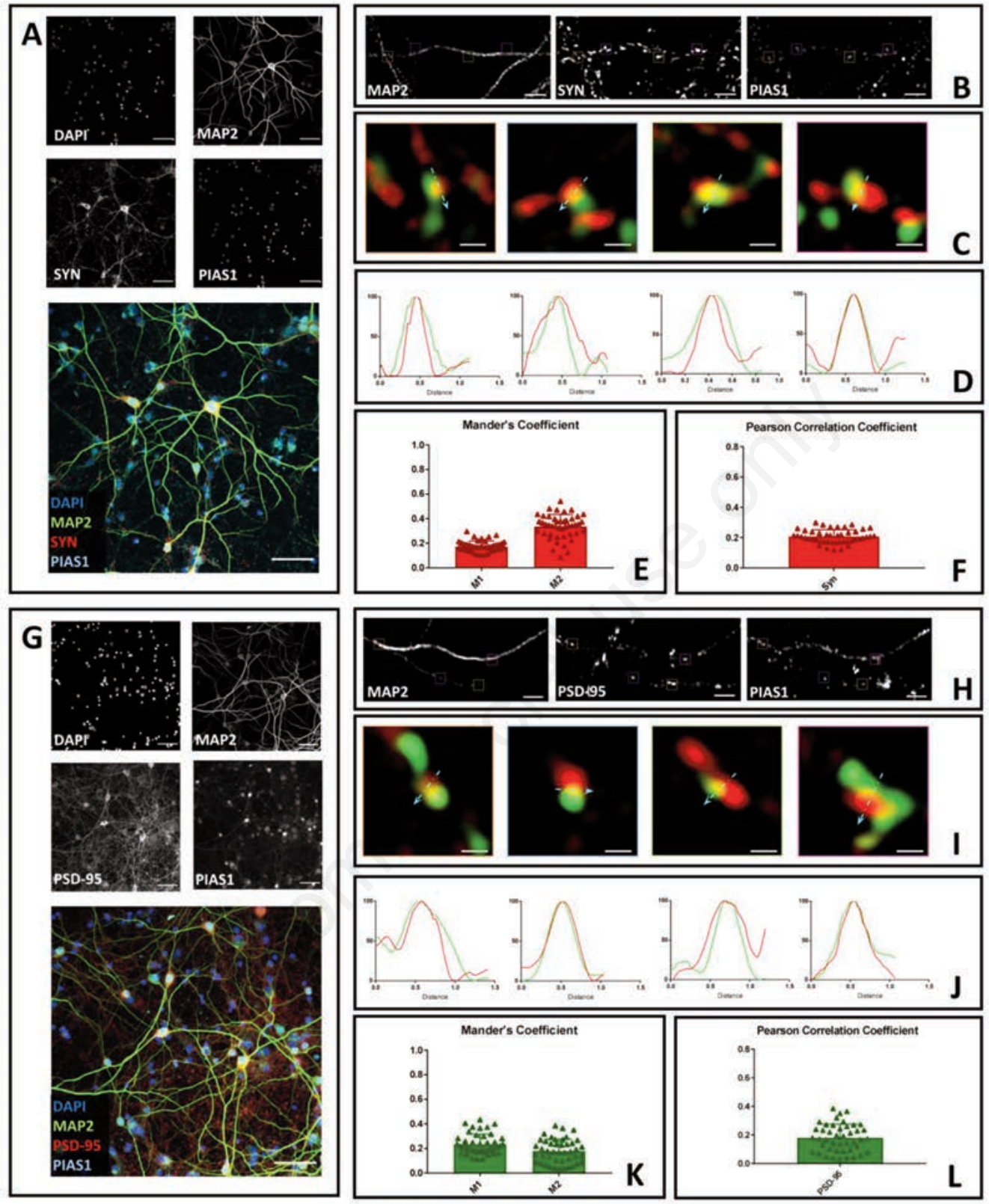

Figure 1. Co-localization of PIAS1 with synaptic markers in hippocampal neurons, by confocal microscopy and SIM. A) The images, obtained using a Nikon N-SIM confocal microscope with a 40x objective, represent neurons treated with antibodies directed against PIAS1 (cyan), synaptophysin (red) and Map2 (green), with a Hoechst stain for the nuclei (blue); scale bar: $50 \mu \mathrm{m}$. B) Analysis of 3DSIM images acquired using a 100x objective, $2 \mu \mathrm{m}$ scale; the colored squares indicate the details presented in (C). C) Merge images showing co-localization between PIAS1 (green) and synaptophysin (red); scale bar: $0.5 \mu \mathrm{m}$. D) Intensity profile, with the values normalized to 100 (arbitrary units), representing the co-localization between PIAS1 (green) synaptophysin (red) indicated by the blue arrow (C). E) Mander's coefficients indicate the PIAS1 fraction that co-localizes with synaptophysin (M1) and the synaptophysin fraction that co-localizes with PIAS1 (M2). F) The Pearson correlation coefficients for PIAS1 and synaptophysin (SYN) data are the mean \pm SD of 40 fields from four independent experiments. G) The images, obtained using Nikon N-SIM confocal microscope with a $40 x$ objective, represent neurons treated with antibodies directed against PIAS1 (cyan), PSD-95 (red) and Map2 (green), with a Hoechst stain for the nuclei (blue); scale bar: $50 \mu \mathrm{m}$. H) Analysis of 3D-SIM images acquired using a 100x objective, $2 \mu \mathrm{m}$ scale; the colored squares indicate the details presented in (I). I) Merge images showing co-localization between PIAS1 and (green) and PSD-95 (red); scale bar: $0.5 \mu \mathrm{m}$. J) Intensity profiles, normalized to 100 (arbitrary units), represent the co-localization between PIAS1 (green) PSD95 (red) indicated by the light blue arrow (I). K) Mander's coefficients indicate the PIAS1 fraction that co-localizes with PSD-95 (M1) and the PSD-95 fraction that co-localizes with PIAS1 (M2). L) The Pearson correlation coefficients of PIAS1 and PSD-95 data are the mean \pm SD of 40 fields from four independent experiments. 
tion, ${ }^{34}$ similarly to its extensively studied phosphorylation. ${ }^{58,59}$ PIAS3 too appears to be involved in learning processes, memory formation, and underlying long-lasting synaptic plasticity. ${ }^{35} \mathrm{nNOS}$, which has a key role in LTP, is SUMOylated by PIAS3 in the hippocampus and this modification is required for hippocampal LTP induction. ${ }^{35}$ That PIAS1 and PIAS3 are involved in the physiology
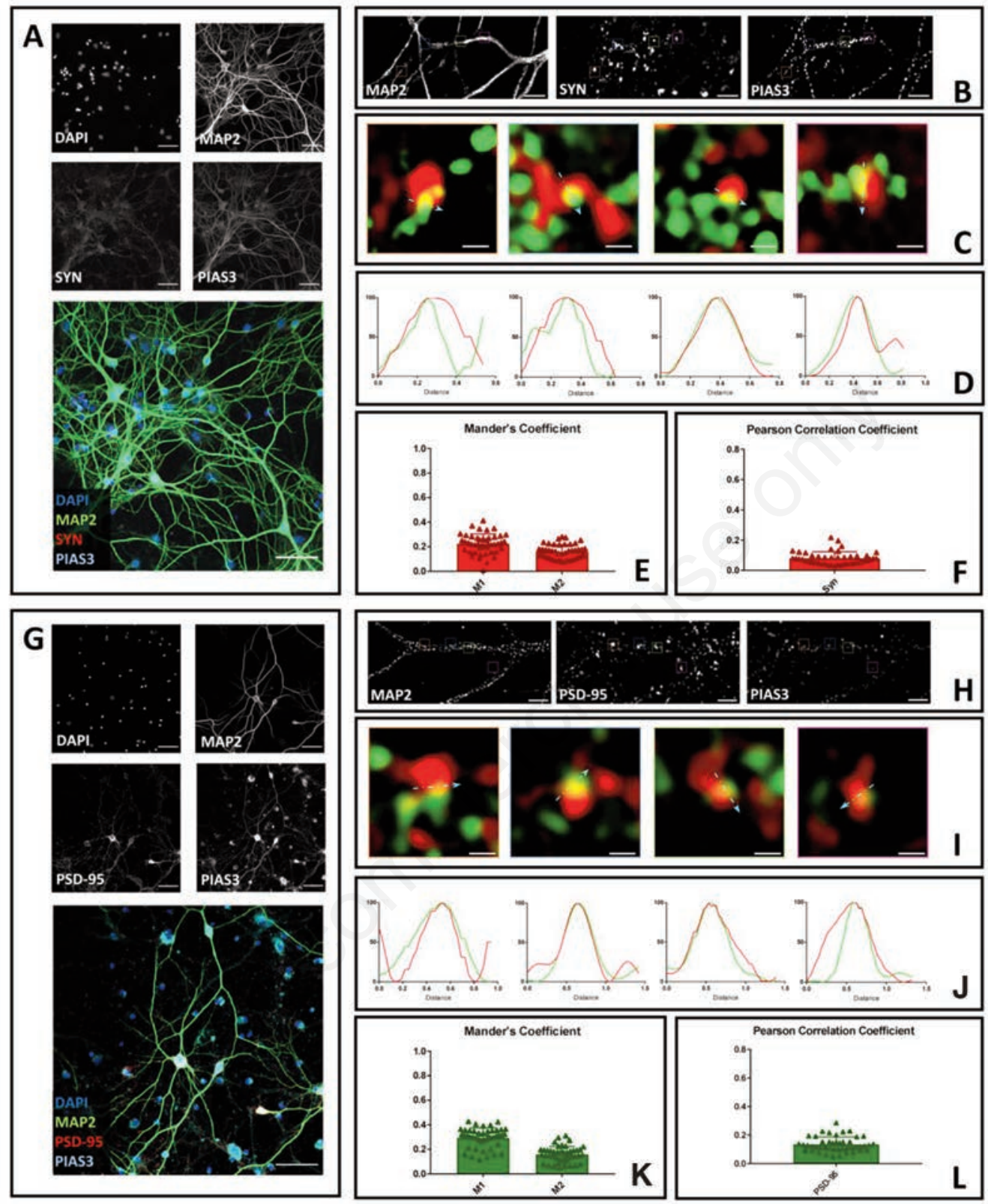

Figure 2. Co-localization of PIAS3 with synaptic markers in hippocampal neurons, by confocal microscopy and SIM. A) The images, obtained using a Nikon N-SIM confocal microscope with a 40x objective, represent neurons treated with antibodies directed against PIAS3 (cyan) synaptophysin (red) and Map2 (green) and a Hoechst stain for the nuclei (blue); scale bar: $50 \mu \mathrm{m}$. B) 3D-SIM images acquired using a 100x objective, $2 \mu \mathrm{m}$ scale; the colored squares indicate the details presented in (C). C) Merge images showing colocalization between PIAS3 (cyan), Map2 (green) and synaptophysin (red); scale bar: $0.5 \mu \mathrm{m}$. D) Intensity profiles, normalized to 100 (arbitrary units), represent the co-localization between PIAS3 (green) and synaptophysin (red) indicated by the light blue arrow (C). E) Mander's coefficients indicate the PIAS3 fraction that co-localizes with synaptophysin (M1) and synaptophysin fraction that colocalizes with PIAS3 (M2). F) Pearson correlation coefficients of PIAS3 and synaptophysin (SYN) are the mean \pm SD of 40 fields from four independent experiments. G) The images, obtained using a Nikon N-SIM confocal microscope with a 40x objective, represent neurons treated with antibodies directed against PIAS3 (cyan), PSD-95 (red) and Map2 (green) and with a Hoechst stain for the nuclei (blue); scale bar: $50 \mu \mathrm{m} . \mathrm{H}$ ) Analysis of 3D-SIM images acquired using a 100x objective, $2 \mu \mathrm{m}$ scale; the colored squares indicate the details presented in (I). I) Merge images showing co-localization between PIAS3 (green) and PSD-95 (red); scale bar: 0.5 $\mu \mathrm{m}$. J) Intensity profiles normalized to 100 (arbitrary units), representing the co-localization between PIAS3 (green) and PSD-95 (red) indicated by the light blue arrow (I). K) Mander's coefficients indicate the PIAS3 fraction that co-localizes with PSD-95 (M1) and the PSD95 fraction that co-localizes with PIAS3 (M2). L) Pearson correlation coefficients of PIAS3 and PSD-95 are the mean \pm S D of 40 fields from four independent experiments. 
of memory is further supported by evidence that their dysfunctions may cause spatial memory difficulties in different neurodegenerative diseases, such as Huntington's disease or Rett syndrome..$^{39,60,61}$
The modulation of synaptic plasticity in cortical neurons by the SUMOylation machinery has been less explored. While in cortical neurons SUMOylation can be induced by external stimuli — for
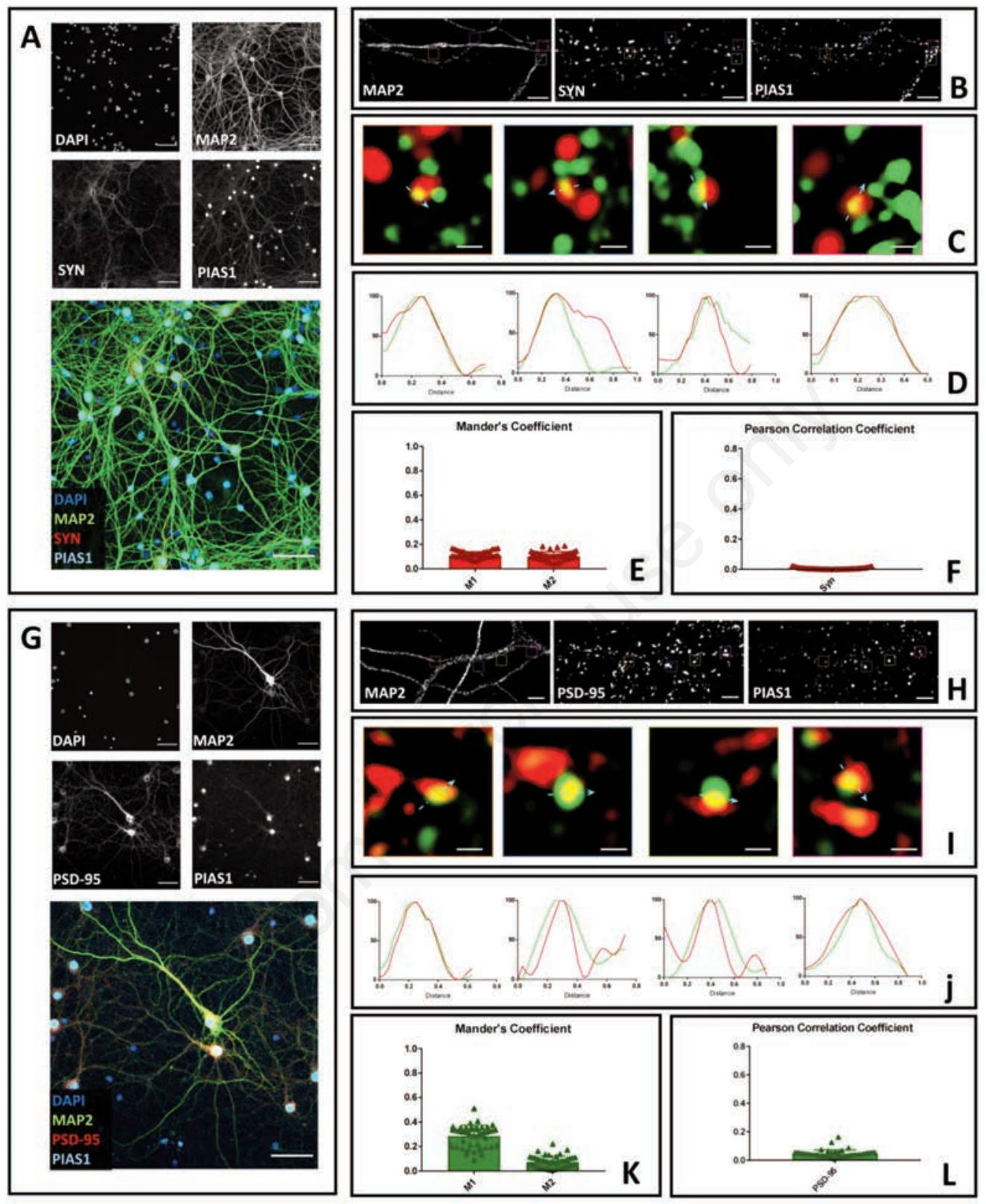

Figure 3. Co-localization of PIAS1 with synaptic markers in cortex neurons through Confocal and SIM microscopy. A) The images, obtained using Nikon N-SIM confocal microscope with a 40x objective, represent neurons treated with antibodies directed against PIAS1 (cyan), synaptophysin (red) and Map2 (green) and with a solution of Hoechst to stain the nuclei (blue); scale bar: $50 \mu \mathrm{m}$. B) Analysis of 3D-SIM images acquired using a 100x objective, $2 \mu \mathrm{m}$ scale; the colored squares indicate the details represented in (C). C) Merge images showing co-localization between PIAS1 and (green) and synaptophysin (red); scale bar: $0.5 \mu \mathrm{m}$. (D) Intensity profile, whose values have been normalized to 100 (arbitrary unit), representing the co-localization between PIAS1 (green) synaptophysin (red) indicated by the light blue arrow (C). E) Mander's coefficients indicate PIAS1 fraction that colocalizes with synaptophysin (M1) and synaptophysin fraction that colocalizes with PIAS1 (M2). F) Pearson Correlation Coefficient of PIAS1 and synaptophysin (SYN) data are the mean \pm SD of 40 fields from four independent experiments. G) The images, obtained using Nikon N-SIM confocal microscope with a 40x objective, represent neurons treated with antibodies directed against PIAS1 (cyan), PSD-95 (red) and Map2 (green) and with a solution of Hoechst to stain the nuclei (blue); scale bar: $50 \mu \mathrm{m} . \mathrm{H}$ ) Analysis of 3D-SIM images acquired using a 100x objective, 2 $\mu \mathrm{m}$ scale; the colored squares indicate the details represented in (I). I) Merge images showing co-localization between PIAS1 (green) and PSD-95 (red); scale bar: $0.5 \mu \mathrm{m}$. J) Intensity profile, whose values have been normalized to 100 (arbitrary unit), representing the co-localization between PIAS1 (green) PSD-95 (red) indicated by the light blue arrow (I). K) Mander's coefficients indicate PIAS1 fraction that colocalizes with PSD-95 (M1) and PSD-95 fraction that colocalizes with PIAS1 (M2). L) Pearson Correlation Coefficient PIAS1 and PSD-95 data are the mean \pm SD of 40 fields from four independent experiments. 
instance guanosine, a purine nucleoside linked to cell metabolism and stress, increases the conjugation of SUMO2/362 - a direct role of the SUMOylation machinery in neuronal activity has not been described. This agrees with evidence that SUMO1-3 knock-down mice appear to have deficits in episodic memory processes and fear learning, phenotypes mediated by the hippocampus and amygdala and to a lesser extent cortical regions. ${ }^{63}$ In cortical neurons, in fact, we found that the co-localization of PIAS1 and PIAS3 with synaptic markers was rare, thus possibly suggesting different roles of the E3 ligases in the two neuronal populations.
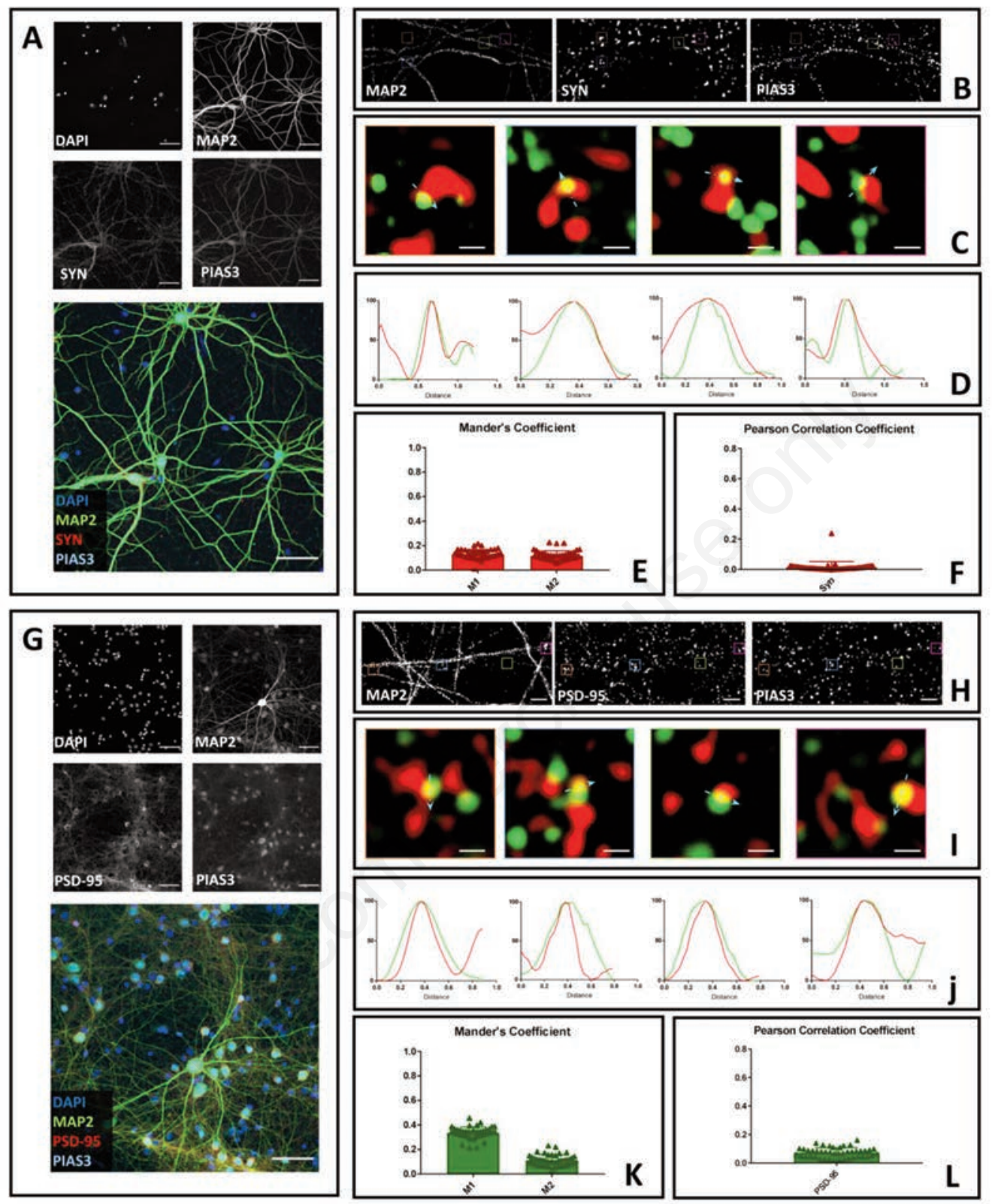

Figure 4. Co-localization of PIAS3 with synaptic markers in cortex neurons, by confocal microscopy and SIM. A) The images, obtained using a Nikon N-SIM confocal microscope with a 40x objective, represent neurons treated with antibodies directed against PIAS3 (cyan), synaptophysin (red), Map2 (green) and a Hoechst stain for the nuclei (blue); scale bar: $50 \mu \mathrm{m}$. B) Analysis of 3D-SIM images acquired using a 100x objective, $2 \mu \mathrm{m}$ scale; the colored squares indicate the details presented in (C). C) Merge images showing colocalization between PIAS3 (green) and synaptophysin (red); scale bar: $0.5 \mu \mathrm{m}$. D) Intensity profiles were normalized to 100 (arbitrary units), representing the co-localization between PIAS3 (green) synaptophysin (red) indicated by the light blue arrow (C). E) Mander's coefficients indicate the PIAS3 fraction that co-localizes with synaptophysin (M1) and the synaptophysin fraction that co-localizes with PIAS3 (M2). F) Pearson's correlation coefficients of PIAS3 and synaptophysin (SYN) are the mean \pm SD of 40 fields from four independent experiments. G) The images, obtained using Nikon N-SIM confocal microscope with a 40x objective, represent neurons treated with antibodies directed against PIAS3 (cyan), PSD-95 (red) and Map2 (green) and with Hoechst stain for the nuclei (blue); scale bar: $50 \mu \mathrm{m} . \mathrm{H}$ ) Analysis of 3D-SIM images acquired using a 100x objective, $2 \mu \mathrm{m}$ scale; the colored squares indicate the details presented in (I). I) Merge images showing co-localization between PIAS3 and (green) and PSD-95 (red); scale bar: $0.5 \mu \mathrm{m}$. J) Intensity profiles were normalized to 100 (arbitrary units), representing the co-localization between PIAS3 (green) PSD-95 (red) indicated by the light blue arrow (I). K) Mander's coefficients indicate the PIAS3 fraction that co-localizes with PSD-95 (M1) and the PSD-95 fraction that co-localizes with PIAS3 (M2). L) Pearson correlation coefficient PIAS3 and PSD-95 are the mean \pm SD of 40 fields from four independent experiments. 
In all these analyses, cultured hippocampal and cortical neurons were in the resting state, not chemically or electrically stimulated. It is therefore possible that neuronal activation might change the co-localization of PIAS1 or PIAS3 with synaptic markers. ${ }^{21}$ This would be in line with evidence that spatial training using a water maze not only increases PIAS1 expression in the rat hippocampus, ${ }^{32}$ but also significantly raises PIAS1 activity, resulting for instance in SUMOylation of CREB1 $\triangle$ (the short isoform of CREB) in rat CA1 neurons. ${ }^{34}$

In summary, the present data expand our understanding of the localization of components of the SUMO machinery in neurons. In previous studies, we observed the co-localization with synaptic markers of SUMO1, SUMO2/3, the E2 ligase Ubc9 and the SUMO isopeptidases SENP1, SENP6 and SENP7. ${ }^{26,27}$ The current analysis adds information on the E3 ligases PIAS1 and PIAS3 at super-resolution level. This study further supports the role of the SUMOylation machinery in the physiology of memory, suggesting our need to understand it better in the light of the connection with synaptopathies such as AD and Parkinson's disease.

\section{References}

1. Routtenberg A, Rekart JL. Post-translational protein modification as the substrate for long-lasting memory. Trends Neurosci 2005;28:12-9.

2. Tak I-R, Ali F, Dar JS, Magray AR, Ganai BA, Chishti MZ. Chapter 1 - Posttranslational modifications of proteins and their role in biological processes and associated diseases. In: Dar TA, Singh LR, editors. Protein Modificomics. Academic Press; 2019. p. 1-35.

3. Kerscher O. SUMO junction-what's your function? New insights through SUMO-interacting motifs. EMBO Rep 2007;8:550-5.

4. Marcelli S, Ficulle E, Iannuzzi F, Kövari E, Nisticò R, Feligioni M. Targeting SUMO-1ylation contrasts synaptic dysfunction in a mouse model of Alzheimer's Disease. Mol Neurobiol 2017;54:6609-23.

5. Schorova L, Pronot M, Poupon G, Prieto M, Folci A, Khayachi A, et al. The synaptic balance between sumoylation and desumoylation is maintained by the activation of metabotropic mGlu5 receptors. Cell Mol Life Sci 2019;76:3019-31.

6. Henley JM, Seager R, Nakamura Y, Talandyte K, Nair J, Wilkinson KA. SUMOylation of synaptic and synapse-associated proteins: An update. J Neurochem 2021;156:145-61.

7. Schorova L, Martin S. Sumoylation in synaptic function and dysfunction. Front Synaptic Neurosci 2016;8:9.

8. Lee L, Sakurai M, Matsuzaki S, Arancio O, Fraser P. SUMO and Alzheimer's disease. Neuromolecular Med 2013;15:720 36.

9. Eckermann K. SUMO and Parkinson's disease. Neuromolecular Med 2013;15:737-59.

10. Guerra de Souza AC, Prediger RD, Cimarosti H. SUMO-regulated mitochondrial function in Parkinson's disease. J Neurochem 2016;137:673-86.

11. Gareau JR, Lima CD. The SUMO pathway: emerging mechanisms that shape specificity, conjugation and recognition. Nat Rev Mol Cell Biol 2010;11:861-71.

12. Bohren KM, Nadkarni V, Song JH, Gabbay KH, Owerbach D. A M55V polymorphism in a novel SUMO gene (SUMO-4) differentially activates heat shock transcription factors and is associated with susceptibility to type I diabetes mellitus. J Biol Chem 2004;279:27233-8.

13. Henley JM, Craig TJ, Wilkinson KA. Neuronal SUMOylation: Mechanisms, physiology, and roles in neuronal dysfunction.
Physiol Rev 2014;94:1249-85.

14. Gareau JR, Reverter D, Lima CD. Determinants of small ubiquitin-like modifier 1 (SUMO1) protein specificity, E3 ligase, and SUMO-RanGAP1 binding activities of nucleoporin RanBP2. J Biol Chem 2012;287:4740-51.

15. Wilkinson KA, Nakamura Y, Henley JM. Targets and consequences of protein SUMOylation in neurons. Brain Res Rev 2010;64:195-212.

16. Rabellino A, Andreani C, Scaglioni PP. The role of PIAS SUMO E3-ligases in cancer. Cancer Res 2017;77:1542-7.

17. ScienceDirect Topics [Internet]. Ubiquitin Protein Ligase E3 an overview. Accessed: 2020 Dec 24. Available from: https://www.sciencedirect.com/topics/medicine-and-dentistry/ubiquitin-protein-ligase-e3

18. Yang W-S, Campbell M, Kung H-J, Chang P-C. In vitro SUMOylation assay to study SUMO E3 ligase activity. J Vis Exp 2018;131:56629.

19. Kunz K, Piller T, Müller S. SUMO-specific proteases and isopeptidases of the SENP family at a glance. J Cell Sci 2018;131:jcs211904.

20. Martin S, Nishimune A, Mellor JR, Henley JM. SUMOylation regulates kainate-receptor-mediated synaptic transmission. Nature 2007;447:321-5.

21. Loriol C, Khayachi A, Poupon G, Gwizdek C, Martin S. Activity-dependent regulation of the sumoylation machinery in rat hippocampal neurons. Biol Cell 2013;105:30-45.

22. Ficulle E, Sufian MDS, Tinelli C, Corbo M, Feligioni M. Aging-related SUMOylation pattern in the cortex and blood plasma of wild type mice. Neurosci Lett 2018;668:48-54.

23. Castro-Gomez S, Barrera-Ocampo A, Machado-Rodriguez G, Castro-Alvarez JF, Glatzel M, Giraldo M, et al. Specific deSUMOylation triggered by acquisition of spatial learning is related to epigenetic changes in the rat hippocampus. Neuroreport 2013;24:976-81.

24. Daniel JA, Cooper BH, Palvimo JJ, Zhang F-P, Brose N, Tirard M. Analysis of SUMO1-conjugation at synapses. eLif. 2017;6:e26338.

25. Daniel JA, Cooper BH, Palvimo JJ, Zhang F-P, Brose N, Tirard M. Response: Commentary: Analysis of SUMO1-conjugation at synapses. Front Cell Neurosci 2018;12:117.

26. Colnaghi L, Russo L, Natale C, Restelli E, Cagnotto A, Salmona M, et al. Super resolution microscopy of SUMO proteins in neurons. Front Cell Neurosci 2019;13:486.

27. Colnaghi L, Conz A, Russo L, Musi CA, Fioriti L, Borsello T, et al. Neuronal localization of SENP proteins with super resolution microscopy. Brain Sci 2020;10:778.

28. Russo L, Natale C, Conz A, Kelk J, Restelli E, Chiesa R, et al. Super-resolution imaging to study co-localization of proteins and synaptic markers in primary neurons. J Vis Exp 2020;164:e61434.

29. Silveirinha VC, Lin H, Tanifuji S, Mochida S, Cottrell GS, Cimarosti $\mathrm{H}$, et al. CaV2.2 (N-type) voltage-gated calcium channels are activated by SUMOylation pathways. Cell Calcium 2021;93:102326.

30. Chung CD, Liao J, Liu B, Rao X, Jay P, Berta P, et al. Specific inhibition of Stat3 signal transduction by PIAS3. Science 1997;278:1803-5.

31. Liu B, Liao J, Rao X, Kushner SA, Chung CD, Chang DD, et al. Inhibition of Stat1-mediated gene activation by PIAS1. Proc Natl Acad Sci USA 1998;95:10626-31.

32. Tai DJC, Hsu WL, Liu YC, Ma YL, Lee EHY. Novel role and mechanism of protein inhibitor of activated STAT1 in spatial learning. EMBO J 2011;30:205-20.

33. Liu SY, Ma YL, Lee EHY. NMDA receptor signaling mediates the expression of protein inhibitor of activated STAT1 (PIAS1) 
in rat hippocampus. Neuropharmacology 2013;65:101-13.

34. Chen Y-C, Hsu W-L, Ma Y-L, Tai DJC, Lee EHY. CREB SUMOylation by the E3 ligase PIAS1 enhances spatial memory. J Neurosci Off J Soc Neurosci 2014;34:9574-89.

35. Du C-P, Wang M, Geng C, Hu B, Meng L, Xu Y, et al. Activityinduced SUMOylation of neuronal nitric oxide synthase is associated with plasticity of synaptic transmission and extracellular signal-regulated kinase 1/2 signaling. Antioxid Redox Signal 2020;32:18-34.

36. Ghosh H, Auguadri L, Battaglia S, Simone Thirouin Z, Zemoura K, Messner S, et al. Several posttranslational modifications act in concert to regulate gephyrin scaffolding and GABAergic transmission. Nat Commun 2016;7:13365.

37. Chiba T, Yamada M, Sasabe J, Terashita K, Shimoda M, Matsuoka M, et al. Amyloid- $\beta$ causes memory impairment by disturbing the JAK2/STAT3 axis in hippocampal neurons. Mol Psychiatry 2009;14:206-22.

38. Liu S-Y, Ma Y-L, Hsu W-L, Chiou H-Y, Lee EHY. Protein inhibitor of activated STAT1 Ser503 phosphorylation-mediated Elk-1 SUMOylation promotes neuronal survival in APP/PS1 mice. Br J Pharmacol 2019;176:1793-810.

39. O’Rourke JG, Gareau JR, Ochaba J, Song W, Raskó T, Reverter D, et al. SUMO-2 and PIAS1 modulate insoluble mutant huntingtin protein accumulation. Cell Rep 2013;4:36275.

40. Morozko EL, Smith-Geater C, Monteys AM, Pradhan S, Lim RG, Langfelder P, et al. PIAS1 modulates striatal transcription, DNA damage repair, and SUMOylation with relevance to Huntington's disease. Proc Natl Acad Sci USA 2021;118:e2021836118.

41. Rubio MD, Wood K, Haroutunian V, Meador-Woodruff JH. Dysfunction of the ubiquitin proteasome and ubiquitin-like systems in schizophrenia. Neuropsychopharmacology 2013;38:1910-20.

42. Huang Z, Fujiwara K, Minamide R, Hasegawa K, Yoshikawa K. Necdin controls proliferation and apoptosis of embryonic neural stem cells in an oxygen tension-dependent manner. J Neurosci Off J Soc Neurosci 2013;33:10362-73.

43. Lin H-Y, Liu Y-S, Huang C-Y, Cathomas F, Liu K, Wang J, et al. SUMO E3 ligase PIAS1 is a potential biomarker indicating stress susceptibility. Psychoneuroendocrinology 2020;120: 104800 .

44. Floriou-Servou A, von Ziegler L, Stalder L, Sturman O, Privitera M, Rassi A, et al. Distinct proteomic, transcriptomic, and epigenetic stress responses in dorsal and ventral hippocampus. Biol Psychiatry 2018;84:531-41.

45. Cordelieres FP, Bolte S. JACoP v2.0: improving the user experience with co-localization studies. Available from: https://imagejdocu.tudor.lu/_media/plugin/analysis/jacop_2.0/ just_another_colocalization_plugin/jacop_ijconf2008.pdf

46. Wiedenmann B, Franke WW. Identification and localization of synaptophysin, an integral membrane glycoprotein of $\mathrm{Mr}$ 38,000 characteristic of presynaptic vesicles. Cell 1985;41:1017-28.

47. Chen X, Winters C, Crocker V, Lazarou M, Sousa AA,
Leapman RD, et al. Identification of PSD-95 in the postsynaptic density using miniSOG and EM tomography. Front Neuroanat 2018;12:107.

48. Dunn KW, Kamocka MM, McDonald JH. A practical guide to evaluating colocalization in biological microscopy. Am J Physiol Cell Physiol 2011;300:C723-42.

49. Huang B, Bates M, Zhuang X. Super-resolution fluorescence microscopy. Annu Rev Biochem 2009;78:993-1016.

50. Igarashi M, Nozumi M, Wu L-G, Zanacchi FC, Katona I, Barna L, et al. New observations in neuroscience using superresolution microscopy. J Neurosci 2018;38:9459-67.

51. Schikorski T, Stevens CF. Quantitative ultrastructural analysis of hippocampal excitatory synapses. J Neurosci 1997; 17:5858-67.

52. Wasik U, Filipek A. Non-nuclear function of sumoylated proteins. Biochim Biophys Acta 2014;1843:2878-85.

53. Adler J, Parmryd I. Quantifying colocalization by correlation: The Pearson correlation coefficient is superior to the Mander's overlap coefficient. Cytometry A 2010;77:733-42.

54. Dabir S, Kluge A, Dowlati A. The association and nuclear translocation of the PIAS3-STAT3 complex is ligand and time dependent. Mol Cancer Res 2009;7:1854-60.

55. Hsu W-L, Ma Y-L, Hsieh D-Y, Liu Y-C, Lee EH. STAT1 negatively regulates spatial memory formation and mediates the memory-impairing effect of $A \beta$. Neuropsychopharmacology 2014;39:746-58.

56. Mizuno M, Yamada K, Maekawa N, Saito K, Seishima M, Nabeshima T. CREB phosphorylation as a molecular marker of memory processing in the hippocampus for spatial learning. Behav Brain Res 2002;133:135-41.

57. Viola H, Furman M, Izquierdo LA, Alonso M, Barros DM, de Souza MM, et al. Phosphorylated cAMP response elementbinding protein as a molecular marker of memory processing in rat hippocampus: effect of novelty. J Neurosci 2000;20:RC112.

58. Silva AJ, Kogan JH, Frankland PW, Kida S. CREB and memory. Annu Rev Neurosci 1998;21:127-48.

59. Kandel ER. The molecular biology of memory: cAMP, PKA, CRE, CREB-1, CREB-2, and CPEB. Mol Brain 2012;5:14.

60. Steffan JS, Agrawal N, Pallos J, Rockabrand E, Trotman LC, Slepko N, et al. SUMO modification of Huntingtin and Huntington's disease pathology. Science 2004;304:100-4.

61. Tai DJC, Liu YC, Hsu WL, Ma YL, Cheng SJ, Liu SY, et al. MeCP2 SUMOylation rescues Mecp2-mutant-induced behavioural deficits in a mouse model of Rett syndrome. Nat Commun 2016;7:10552.

62. Zanella CA, Tasca CI, Henley JM, Wilkinson KA, Cimarosti HI. Guanosine modulates SUMO2/3-ylation in neurons and astrocytes via adenosine receptors. Purinergic Signal 2020;16:439-50.

63. Wang L, Rodriguiz RM, Wetsel WC, Sheng H, Zhao S, Liu X, et al. Neuron-specific Sumo1-3 knockdown in mice impairs episodic and fear memories. J Psychiatry Neurosci 2014;39:259-66. 